\title{
The software methodology to the soft targets assessment
}

\author{
Lucia Mrazkova Duricova ${ }^{1 *}$, Pavel Valasek $^{2}$, Jan Mrazek $^{3}$, Hana Chudá $^{4}$ \\ ${ }^{1234}$ Faculty of Applied Informatics, Tomas Bata University in Zlin, Nad Stranemi 4511, 76005, Zlin, Czech Republic
}

\begin{abstract}
The soft targets and crowded places are closely related to the risk of attack on a group of people. These places are very specific because the moving in the soft targets is not organized. That's mean these places have open access and the public. The attack in the soft targets (attack on the soft targets) can have a significant impact on the population and life of the people. The main aim of the proposed software is to analyze the features of the object. According to the analyses, we can define the corrective action, which can have a significant impact on the security situation in the object.
\end{abstract}

\section{Introduction}

The definition of soft targets can have some differences in the Czech Republic and abroad.

Soft Targets and Crowded Places (ST-CPs) are locations that are easily accessible to large numbers of people and that have limited security or protective measures in place making them vulnerable to attack. STCPs can include but are not limited to, schools, sports venues, transportation systems or hubs, shopping venues, bars and restaurants, hotels, places of worship, tourist attractions, theatres, and civic spaces. ST-CPs do not have to be buildings and can include open spaces such as parks and pedestrian malls. ST-CPs will not necessarily be crowded at all times - crowd densities may vary between day and night, by season, and may be temporary, as in the case of sporting events, festivals, or other special events. [1]

Attacks against soft targets have a powerful effect on the psyche of the populace. Modern terrorist groups and actors had redrawn the battlefield lines, and places where civilians once felt secure have been pulled into the war zone. [2]

In this paper, we describe the methodology of the assessment of the soft targets. In section 1, we describe the closed history of the attacks from 2019 . In section 2 , we describe the criteria of the software. The mathematical definition is described in Section3. The case study is described in section 4. Finally, we constant the results of the analyses in the last part of the paper.

\subsection{The attacks on the civilians in soft targets}

The attacks on soft targets are very popular in the last years. The reason for the attacks on the soft targets is that the soft targets are full of unprotected civilians and these places are called "free gun zones". [2]
In Figure 1, you can see the timeline of the attacks in the 2018 .

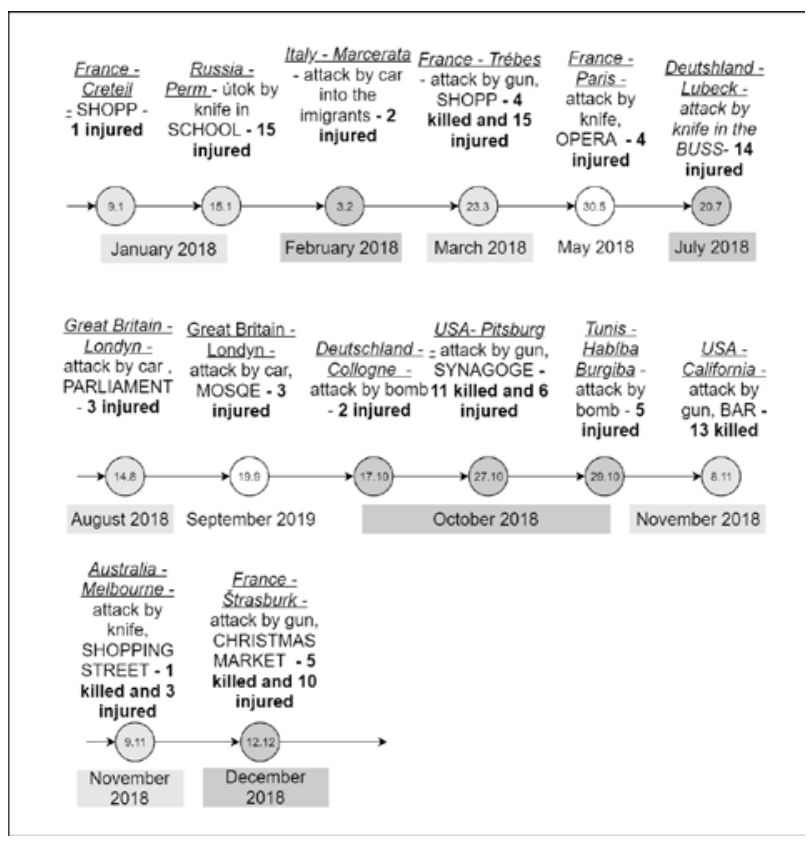

Fig. 1. The timeline of the attacks in 2019.

In Figure 1, we can see the number of killed civilians. The situation in Figure 1 describes the places which can be attacked. According to the HESTERMAN (2015) can be described the similar motivations and goals that cross all brands of the terrorist and criminal group to attack the soft targets:

- Easier, cheaper, and short planning cycle. (The attack on the soft targets is easier, cheaper and short that the attack on the military objects. The effect on the population has a significant impact)

\footnotetext{
* Corresponding author: 1mrazkova@utb.cz
} 
- Increased likelihood of success.

- Credibility. (Status in the group.)

- Recruiting value.

- Flexing muscle.

- Compensating for weakness.

- As a last gasp.

- Backed into the corner.

- Test a new strategy, tactic, or weapon.

- Fund-raising.

- Quickly damage a market.

- Delegitimize a government.

- Cause political instability.

- Make a country look weak internationally.

- To attain global media coverage.

- A target-rich environment.

- Psychological fear.

- Make a domestic issue international.

[2]

In 2019 (21.4.2019) exposes 6 bombs in Sri Lance. This attack can be called the biggest attack in 2019 (up to now). This attack caused 42 deaths and 280 injured civilians.

In this part of the paper was described the attacks on the soft targets. We can constant that this part of the research still needs to innovate and develop the next approaches to solving the situation.

\section{The definition of the criteria of the software}

The criteria of the soft target software tool can be divided into the next categories:

- General basic criteria

- Exterior criteria

- Interior criteria

- Processes criteria

General basic criteria define the general state of the object. The locality is one of the general basic criteria. The locality is defined according to the address of the object (GPS). The map tool has defined the locality security coefficient according to the significance of the map point. The significance of the object or the area can have a significant impact on the probability of the attack on soft targets.

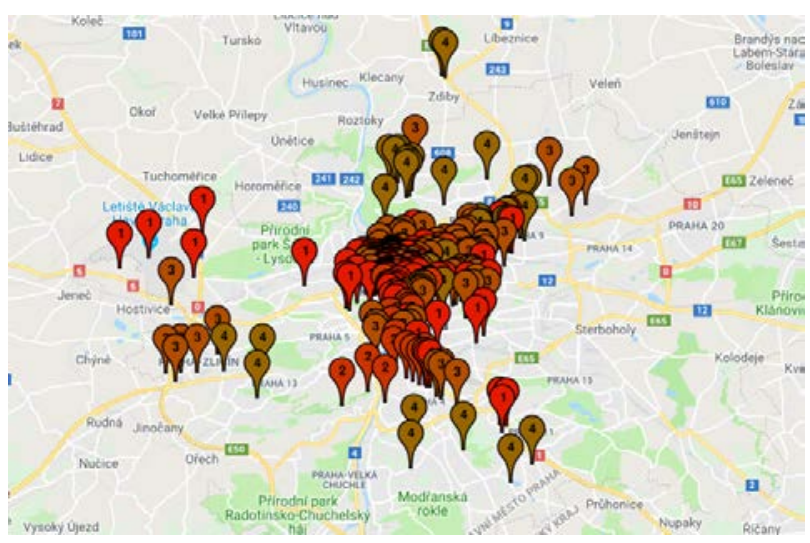

Fig. 2. Map Tool of the Software.

In Figure 2 we can see the Map Tool, which can calculate the security coefficient of the locality. Figure 2 can be seen in the amount of the evaluated points.

One of the next general basic criteria is the number of visitors per day in the object. This amount we can estimate, or calculate according to the population number and attractiveness index, or we can use the modern information technology. The research does not implement these modern technologies because these objects are commercial and the current research doesn't have so many financial resources. The detection of the user's amount according to the linked telephone numbers and the detection according to the linked PC and mobile devices to the Wi-Fi belong to these information technologies.

The last criteria are the categorization of the object. The categorization can have a significant impact on the weight of the criteria and risk of the incident.

\section{The mathematical definition of the software tool}

The software logic is based on the analyses of the features of the building or closed area of the building or event. The level of the features is examined according to the questions and answers. The level of the feature can say us how is level of the risk in the object.

The level of the security can be influenced by the security measures. After the repeated assessment we can see the higher level of the security.

The whole coefficient of the object is defined in the next equations:

$$
\mathrm{K}_{\mathrm{S}}=\frac{\mathrm{L} \cdot \mathrm{W}_{1}+\mathrm{C}_{\mathrm{EK}} \cdot \mathrm{W}_{2}+\mathrm{C}_{\mathrm{PK}} \cdot \mathrm{W}_{3}+\mathrm{C}_{\mathrm{IK}} \cdot \mathrm{W}_{3}}{4}
$$

$\mathrm{K}_{\mathrm{S}}$ - the Final security coefficient

$\mathrm{Wn}$ - weight of the each of coefficient

$\mathrm{L}$ - Coefficient of the Locality

$\mathrm{C}_{\mathrm{EK}}-$ Final Coefficient of the Exterior

$\mathrm{C}_{\mathrm{PK}}$ - Final Coefficient of the Processes

$\mathrm{C}_{\mathrm{IK}}$ - Final Coefficient of the Interior 
The weight $(\mathrm{Wn})$ is set by the administrator of the software tool. We propose that these weights will be clarified after the more case studies. In the current research we evenly set these weights. The locality coefficient is defined by the Map Tool. Map Tool has defined the risk of the locality by the administrator.

The coefficient of the interior is defined in the equation 2. Each of the security attributes can be used in the object several times. The used of these security attributes has significant to the whole Interior security. The security attributes define how much times are the security attributes used.

$$
I_{K}=\frac{1}{P B} \sum_{i=1}^{P B} B_{i},\left(B_{i} \in<0,100\right)
$$

$\mathrm{PB}$ - the number of the security attributes

$\mathrm{Bi}$ - the security attributes

$\mathrm{I}_{\mathrm{K}}$ - the criteria of the Interior

Equation 3 defines the final interior criteria in the same category of the interior. The final interior criteria (category) are based on the sum of the each of these categories interior criteria.

$$
\mathrm{I}_{\mathrm{KCi}}=\frac{1}{\mathrm{PK}} \sum_{\mathrm{i}=1}^{\mathrm{PK}} \mathrm{I}_{\mathrm{K}}
$$

$\mathrm{I}_{\mathrm{KCi}}$ - Interior Criteria (all)

$\mathrm{PK}$ - the number of the criteria

The categories of the interior criteria are incorporated to the Final Coefficient of the Interior. This equation is defined in the equation4.

$$
\mathrm{C}_{\mathrm{IK}}=\frac{1}{\mathrm{P}} \sum_{\mathrm{i}=1}^{\mathrm{P}} \mathrm{I}_{\mathrm{KGi}}
$$

$\mathrm{C}_{\mathrm{IK}}-$ The Final Coefficient of the Interior

$\mathrm{P}-\mathrm{The}$ number of up criteria

The final coefficient of the interior is sum of the all up criteria. The exterior coefficient is defined in the equation 5 .

$$
\mathrm{E}_{\mathrm{K}}=\sum_{\mathrm{k}_{\mathrm{u}}=0}^{3} \mathrm{~N} * \mathrm{k}_{\mathrm{u}}=\mathrm{n} * 0+\mathrm{n} * 1+\mathrm{n} * 2+\mathrm{n} * 3
$$

$\mathrm{E}_{\mathrm{K}}$ - the calculation of the value of the exterior criteria

$\mathrm{k}_{\mathrm{u}}$ - coefficient of the security level

$\mathrm{N}-$ the number of the attributes

$$
\mathrm{E}_{\mathrm{KC}}=\frac{\sum_{\mathrm{ku}=0}^{3} \mathrm{n} * \mathrm{k}_{\mathrm{u}}}{3 * \operatorname{sum} \mathrm{N}}
$$

$\mathrm{E}_{\mathrm{KC}}-$ all of the criteria of the Exterior

Sum $\mathrm{N}$ - the number of all attributes

$$
\operatorname{sum} \mathrm{N}=\sum_{\mathrm{i}=1}^{4} \mathrm{~N}_{\mathrm{i}}
$$

The final coefficient of the exterior is defined in equation 8 .

$$
\mathrm{C}_{\mathrm{EK}}=\left[\frac{10}{\mathrm{n}} \sum_{\mathrm{j}}^{\mathrm{n}} \mathrm{E}_{\mathrm{KCj}}\right] * \mathrm{~W}
$$

$\mathrm{C}_{\mathrm{EK}}$ - the whole coefficient of the Exterior

The each of these equations is used in the next part of the paper (in the case study).

The processes coefficient is defined in equation 9.

$$
\mathrm{P}_{\mathrm{K}}=\sum_{\mathrm{k}_{\mathrm{u}}=1}^{3} \mathrm{n}_{\mathrm{k}} * \mathrm{k}_{\mathrm{u}}
$$

$\mathrm{P}_{\mathrm{K}}$ - coefficient of the one process (the number of criteria)

$\mathrm{n}_{\mathrm{k}}-$ the number of the criteria

$\mathrm{k}_{\mathrm{u}}$ - the level of the criteria

$$
\mathrm{P}_{\mathrm{KC} \mathrm{j}}=\frac{10}{3 * \mathrm{~N}} \sum_{\mathrm{i}=1}^{\mathrm{n}} \mathrm{P}_{\mathrm{Ki}}
$$

$\mathrm{P}_{\mathrm{KCj}}$ - the complete process coefficient of the all processes in one category (processes are divided into the categories)

$\mathrm{N}$ - the number of the processes

$\mathrm{n}$ - the number of the upper level of the process

$\mathrm{P}_{\mathrm{Ki}}-$ the each of the coefficient of the one process

The complete processes coefficient is defined in equation 11. Each categories has defined the weight according to the threats or we can the weight evenly set.

$$
\mathrm{C}_{\mathrm{PK}}=\left[\frac{1}{\mathrm{~N}} \sum_{\mathrm{j}=1}^{\mathrm{k}} \mathrm{P}_{\mathrm{KC} \mathrm{j}}\right] \mathrm{W}
$$

$\mathrm{C}_{\mathrm{PK}}$ - the whole coefficient of the Processes

$\mathrm{k}$ - the criteria

$\mathrm{W}$ - the weight of the process

This part of the chapter defined the mathematical definitions of the whole process of the analysis. We have defined three types of concrete analysis (processes, internal and external) and one type of outside analysis (locality coefficient). The locality coefficient is defined according to the situation in the nearest area of the object. We can say that the locality can be changer in time without the change in the object. For example, the public event can influence the security situation in the object (for example the Christmas market).

\section{Case studies}


The case study is oriented to compare the bus and train station analyses and shopping centers analyses. All objects are oriented in Czech and Slovak republic.

\subsection{The bus and train stations analyses}

The case study of the train and bus stations is based on the analyses of the 7 objects in the Czech Republic. The final data of the analyses we can see in Figure 3.

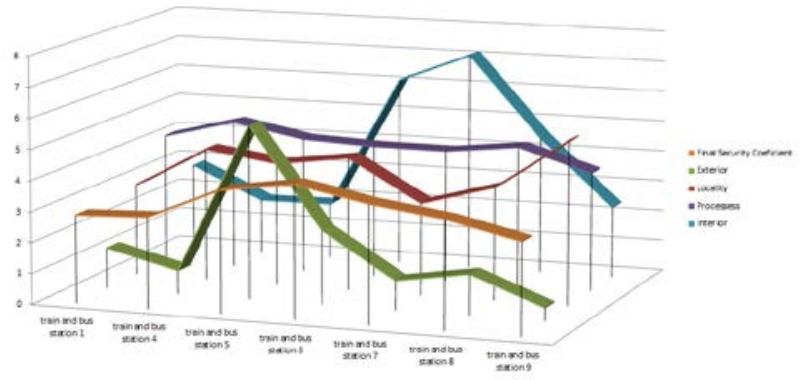

Fig. 3. The bus and train station analyses.

As you can see in Figure 3, the Final security coefficient of these objects is between 2.85 to 4.30 . We can constant that the coefficient is very low because these objects are permanently open and the security measures are very impossible to integrate into the process.

Table 1. The Final Security Coefficient of the Bus and Train Stations

\begin{tabular}{|c|c|}
\hline Object & $\mathbf{K}_{\mathbf{S}}$ \\
\hline $\mathbf{1}$ & $\mathbf{2 . 8 5}$ \\
\hline 4 & 2.94 \\
\hline 5 & 3.96 \\
\hline $\mathbf{6}$ & $\mathbf{4 . 3}$ \\
\hline 7 & 3.83 \\
\hline 8 & 3.51 \\
\hline 9 & 2.98 \\
\hline
\end{tabular}

The average of the KS coefficient is 3.48. As you can see in Table 1, the best security situation is in the object 6. This object is only a small bus station in the small village. The risk of the attack in this object is very low. On the other hand, object number 1 is the object in the county town. The risk of the attack is higher than in the object.

\section{The shopping centres analyses}

The shopping centers are objects with a significantly higher level of security. These objects are commercial use and the security is the aim of the owner and users too. In Figure 4 we can see the results of the analyses.

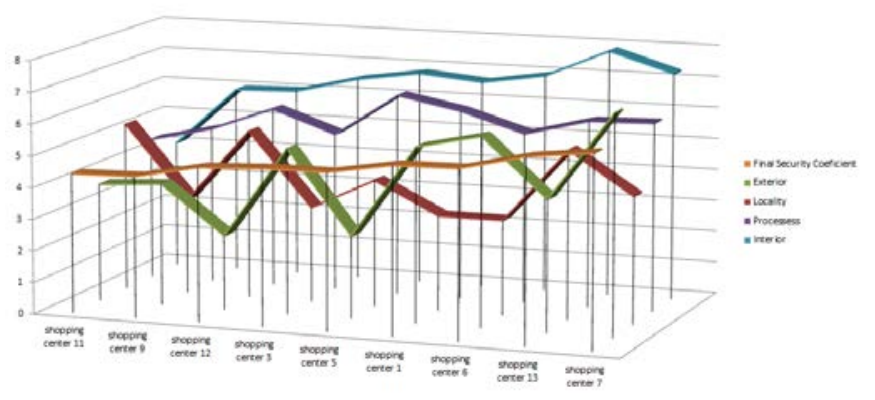

Fig. 4. The shopping centres analyses.

The average value of the analysis is 5.02. The KS is between values 4.03 to 7.21. As you can see in Table 2, the worst security situation is in object 4 . This object is in the middle of the country town. On the other hand, the best security situation is in the object which is oriented out of the country town. The construction predisposition of object number 10 is better than in the object 4 .

Table 2. The Final Security Coefficient of the Shopping centres

\begin{tabular}{|c|c|}
\hline Object & $\mathbf{K}_{\mathbf{S}}$ \\
\hline $\mathbf{4}$ & $\mathbf{4 . 0 3}$ \\
\hline 8 & 4.39 \\
\hline 11 & 4.40 \\
\hline 9 & 4.44 \\
\hline 12 & 4.85 \\
\hline 3 & 4.94 \\
\hline 5 & 4.95 \\
\hline 1 & 5.29 \\
\hline 6 & 5.32 \\
\hline 13 & \\
\hline 1 & \\
\hline
\end{tabular}




\begin{tabular}{|c|c|}
\hline 7 & 6.03 \\
\hline 2 & 6.05 \\
\hline 10 & 7.21 \\
\hline
\end{tabular}

Finally, we can constant that the proposed analyses correspond to reality. The bus and train stations have significantly lowered the security coefficient as the shopping centers.

\section{Conclusions}

Finally, we can constant that the software tool can have better criteria to the study open spaces. The criteria are more oriented to the analyses of the closed objects. For example shopping centers, schools, theatres, and others.

This paper is realized as the doctoral student research. This work was supported by the Internal Grant Agency of Tomas Bata University in Zlin under the project No. IGA/FAI/2019/009 and by the research project VI20172019073 "Identification and methods of protection of Czech soft targets against violent acts with elaboration of a warning system", supported by the Ministry of the Interior of the Czech Republic in the years 2017-2019 and by the research project VI20172019054 "An analytical software module for the real-time resilience evaluation from point of the converged security ", supported by the Ministry of the Interior of the Czech Republic in the years 2017-2019.

\section{References}

1. U.S. Department of Homeland Security, Soft Targets and Crowded Places Security Plan Overview., (2018)

2. Hesterman J., Soft target hardening: protecting people from attack. Boca Raton: CRC Press, Taylor \& Francis Group, 2015, xxii, 299. ISBN 978-14822-4421-2, (2015)

3. Ministry of the Interior Czech Republic, The basics of the protection the soft targets - metodics, (2016)

4. Rosenberg, F.: Nice Solutions for Critical Facilities, Nidam, NICE, (2014)

5. Fekete, A. Safety and Security Target Levels: Opportunities and Challenges for Risk Management and Risk Communication, International Journal of Disaster Risk Reduction 2: 67-76, (2012) 